\section{Lipidomic analysis}

\author{
Michal Holčapek ${ }^{1}$ (D) $\cdot$ Gerhard Liebisch $^{2} \cdot$ Kim Ekroos $^{3}$
}

Published online: 27 January 2020

(C) Springer-Verlag GmbH Germany, part of Springer Nature 2020

The growth of the field of lipidomic analysis is enormous with numerous papers published annually, as shown on the statistics from Web of Science for keywords "lipidomic(s)" and "mass spectrometry" (Fig. 1) with 2923 published papers, where over a hundred of them have been published in Analytical and Bioanalytical Chemistry. The detailed analysis of these papers shows the approximate relative portions for major analytical approaches in lipidomic analysis: 59\% liquid chromatography, 29\% shotgun (direct infusion), and $12 \%$ mass spectrometry imaging. The following authors have contributed the most frequently to this set: X.L. Han (99), G. Liebisch (52), P.J. Meikle (50), R.W. Gross (44), M. Oresic (44), and M.R. Wenk (44). We also highlight five most cited papers from this set [1-5].

In 2015, Analytical and Bioanalytical Chemistry published a previous topical collection on lipidomics [6]. Lipidomic analysis is almost exclusively based on mass spectrometry (MS) analysis [7], starting from low-

Published in the topical collection Current Progress in Lipidomics with guest editors Michal Holčapek, Gerhard Liebisch, and Kim Ekroos.

Michal Holčapek

Michal.Holcapek@upce.cz

Gerhard Liebisch

Gerhard.Liebisch@klinik.uni-regensburg.de

Kim Ekroos

kim@lipidomicsconsulting.com

1 Department of Analytical Chemistry, Faculty of Chemical Technology, University of Pardubice, Studentská 573, 53210 Pardubice, Czech Republic

2 Institute of Clinical Chemistry and Laboratory Medicine, University Hospital Regensburg, 93053 Regensburg, Germany

3 Lipidomics Consulting Ltd., 02230 Espoo, Finland resolution analyzers (typical QqQ), now shifting more towards tandem mass analyzers with ultrahigh resolving power, e.g., Orbitraps. New trends are the implementation of ion mobility, dedicated approaches for the determination of double-bond positions, and ultrahighperformance separation techniques to speed up the analysis time, such as ultrahigh-performance liquid chromatography and ultrahigh-performance supercritical fluid chromatography coupled with MS. Current challenges in the field of lipidomics include high variations in concentrations as well as misquantification and misidentifications [8]. Therefore, Lipidomics Standards Initiative (LSI) [9] was recently founded to develop guidelines for lipidomics. These efforts are aligned with other major initiatives in the field, such as Lipid MAPS [10]. Moreover, the International Lipidomic Society (ILS) [11] just started to represent and promote lipidomics through worldwide cooperation also by hosting various interest groups like LSI or the development of reference values for human plasma [12]. These activities are embedded in a series of conferences, providing space and time to engage with interest groups and discuss further developments.

This topical collection on Lipidomic Analysis includes research papers and one review from leading lipidomic groups worldwide, which represent latest advances in lipidomics and a variety of methods, such as shotgun, LC, and SFC coupled to low- and highresolution mass spectrometry, in line with the current challenges. We express our gratitude to all authors for their excellent contributions, as well as the reviewers, and editorial office for their great efforts to compile this topical collection. 
Fig. 1 The annual number of published papers for keywords "lipidomic(s)" and "mass spectrometry" according to the Web of Science search from December 15, 2019



20032004200520062007200820092010201120122013201420152016201720182019

\section{References}

1. Wenk MR. The emerging field of lipidomics. Nat Rev Drug Discov. 2005;4(7):594-610 (733 citations).

2. Han XL, Gross RW. Shotgun lipidomics: electrospray ionization mass spectrometric analysis and quantitation of cellular lipidomes directly from crude extracts of biological samples. Mass Spectrom Rev. 2005;24(3):367-412 (733 citations).

3. Matyash V, Liebisch G, Kurzchalia TV, Shevchenko A, Schwudke D. Lipid extraction by methyl-tert-butyl ether for high-throughput lipidomics. J Lipid Res. 2008;49(5):1137-46 (700 citations).

4. Quehenberger O, Armando AM, Brown AH, Milne SB, Myers DS, Merrill $\mathrm{AH}$, et al. Lipidomics reveals a remarkable diversity of lipids in human plasma. J Lipid Res. 2010;51(11):3299-305 (586 citations).

5. Ejsing CS, Sampaio JL, Surendranath V, Duchoslav E, Ekroos K, Klemm RW, et al. Global analysis of the yeast lipidome by quantitative shotgun mass spectrometry. Proc Natl Acad Sci U S A. 2009;106(7):2136-41 482 citations.

6. Holčapek M. Lipidomics. Anal Bioanal Chem. 2015;407(17): 4971-2.

7. Holčapek M, Liebisch G, Ekroos K. Lipidomic analysis. Anal Chem. 2018;90(7):4249-57.

8. Liebisch G, Ahrends R, Arita M, Arita M, Bowden JA, Ejsing CS, et al. Lipidomics needs more standardization. Nature Metabolism. 2019;1(8):745-7.

9. Lipidomics Standard Initiative website, https://lipidomicsstandards-initiative.org/

10. Lipid MAPS website, http://www.lipidmaps.org/

11. International Lipidomic Society website, https://ipidomicssociety. org/

12. Burla B, Arita M, Arita M, Bendt AK, Cazenave-Gassiot A, Dennis EA, et al. MS-based lipidomics of human blood plasma: a community-initiated position paper to develop accepted guidelines. J Lipid Res. 2018;59(10):2001-17.

Publisher's note Springer Nature remains neutral with regard to jurisdictional claims in published maps and institutional affiliations.

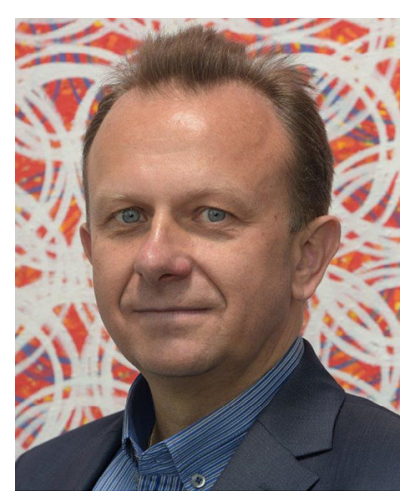

Michal Holčapek obtained his Ph.D. at the Department of Analytical Chemistry, University of Pardubice. Now he is a full professor of analytical chemistry at the same university. His research focus is in mass spectrometry and its coupling with liquid chromatography and supercritical fluid chromatography, applied mainly in the lipidomic analysis and cancer biomarker research. $\mathrm{He}$ is a co-author of over 130 papers in peer-reviewed international journals, h-index 39 , and a member of the editorial advisory boards of Analytical and Bioanalytical Chemistry and Lipids. He was a co-editor of the books Handbook of Advanced Chromatography/Mass Spectrometry Techniques (Academic Press and AOCS Press, 2017) and Extreme Chromatography: Faster, Hotter, Smaller (American Oil Chemical Society, 2011). He is one of founding members of the Lipidomics Standards Initiative and Vice President for conferences in the International Lipidomics Society.

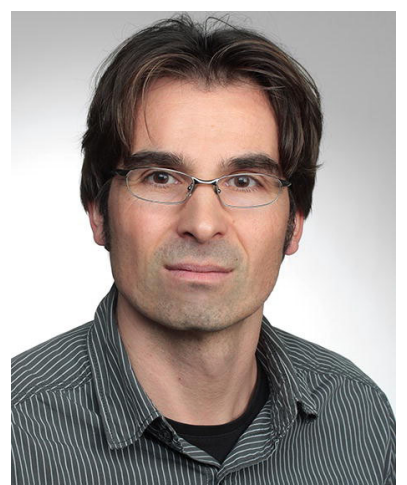

Gerhard Liebisch obtained his Ph.D. at the University of Regensburg. He is a research associate at the Institute of Clinical Chemistry and Laboratory Medicine at the University Hospital in Regensburg and responsible for the instrumental analytics lab. His research interests focus on the development of mass spectrometric methods for lipid species quantification. For more than 20 years, these methods have been applied in clinical studies and basic research, including the use of stable isotope-labelled lipid(s)/precursor to trace transport and metabolism of lipids. He is a co-author of more than 190 papers in peerreviewed international journals, h-index 43, and an editorial board member of The Journal of Lipid Research. He is a co-founder of the Lipidomics Standards Initiative and a board member of the International Lipidomics Society. 


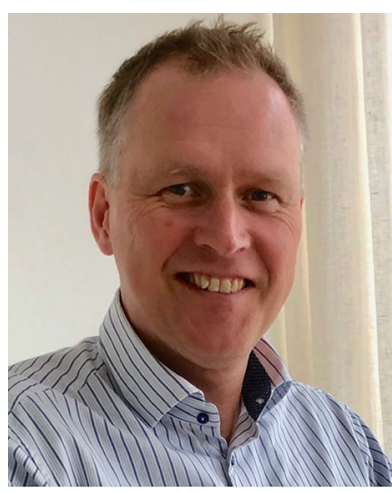

Kim Ekroos, PhD, is the founder and CEO of Lipidomics Consulting Ltd. His expertise includes high-throughput technologies for the precise assessment of lipidomes enabled by advanced mass spectrometry, automation, and software tools towards discovery of biological architectures and of diagnostic biomarkers for clinical purpose. He is one of the pioneers in the field of lipidomics, with $>20$ years of experience in the academic, industry, and regulatory disciplines of lipidomics.

$\mathrm{He}$ is a co-founder of the Lipidomics Standards Initiative (LSI) and President of the International Lipidomics Society. 